\title{
Uncovering of major genetic factors generating naturally occurring variation in heading date among Asian rice cultivars
}

\author{
Kaworu Ebana - Taeko Shibaya · Jianzhong Wu $\cdot$ Kazuki Matsubara $\cdot$ Hiroyuki Kanamori \\ Hiroko Yamane · Utako Yamanouchi - Tatsumi Mizubayashi • Izumi Kono • Ayahiko Shomura • \\ Sachie Ito $\cdot$ Tsuyu Ando $\cdot$ Kiyosumi Hori $\cdot$ Takashi Matsumoto $\cdot$ Masahiro Yano
}

Received: 3 September 2010/ Accepted: 11 December 2010/Published online: 13 January 2011

(C) The Author(s) 2011. This article is published with open access at Springerlink.com

\begin{abstract}
To dissect the genetic factors controlling naturally occurring variation of heading date in Asian rice cultivars, we performed QTL analyses using $\mathrm{F}_{2}$ populations derived from crosses between a japonica cultivar, Koshihikari, and each of 12 cultivars originating from various regions in Asia. These 12 diverse cultivars varied in
\end{abstract}

Communicated by E. Carbonell.

K. Ebana and T. Shibaya contributed equally to this work.

Nucleotide sequences from 12 accession are deposited in DNA Data Bank of Japan as Hdl (AB564428-AB564439), Hd3a (AB564440AB564451), RFT1 (AB564452-AB564463) and Ghd7 (AB564464AB564474).

Electronic supplementary material The online version of this article (doi:10.1007/s00122-010-1524-1) contains supplementary material, which is available to authorized users.

K. Ebana · T. Shibaya $\cdot$ K. Matsubara - U. Yamanouchi .

K. Hori · M. Yano $(\bowtie)$

QTL Genomics Research Center, National Institute of

Agrobiological Sciences, 2-1-2 Kannondai, Tsukuba, Ibaraki

305-8602, Japan

e-mail: myano@nias.affrc.go.jp

J. Wu $\cdot$ T. Matsumoto

Plant Genome Research Unit,

National Institute of Agrobiological Sciences,

2-1-2 Kannondai, Tsukuba, Ibaraki 305-8602, Japan

H. Kanamori · H. Yamane - T. Mizubayashi · I. Kono ·

A. Shomura $\cdot$ S. Ito $\cdot$ T. Ando

Institute of Society for Techno-innovation of Agriculture,

Forestry and Fisheries, Tsukuba, Ibaraki 305-0854, Japan

Present Address:

K. Matsubara

National Institute of Crop Science,

2-1-18 Kannondai, Tsukuba, Ibaraki 305-8518, Japan heading date under natural field conditions in Tsukuba, Japan. Transgressive segregation was observed in $10 \mathrm{~F}_{2}$ combinations. QTL analyses using multiple crosses revealed a comprehensive series of loci involved in natural variation in flowering time. One to four QTLs were detected in each cross combination, and some QTLs were shared among combinations. The chromosomal locations of these QTLs corresponded well with those detected in other studies. The allelic effects of the QTLs varied among the cross combinations. Sequence analysis of several previously cloned genes controlling heading date, including $H d 1, H d 3 a, H d 6, R F T 1$, and $G h d 7$, identified several functional polymorphisms, indicating that allelic variation at these loci probably contributes to variation in heading date. Taken together, the QTL and sequencing results indicate that a large portion of the phenotypic variation in heading date in Asian rice cultivars could be generated by combinations of different alleles (possibly both loss- and gain-of-function) of the QTLs detected in this study.

\section{Introduction}

Heading date is one of crucial factors determining regional and seasonal adaptation in rice and has been a major target of selection in breeding programs. Heading date is a complex trait that is governed by multiple genes and environmental factors, such as daylength, temperature, and soil conditions. A wide range of variation in heading date has been observed among rice cultivars. During the last decade, genetic studies using DNA markers have facilitated the genetic dissection of heading date, and many quantitative trait loci (QTLs) for heading date have been identified using several mapping populations (reviewed by Yano et al. 2001; Hittalmani et al. 2003; Lin et al. 2002, 2003; 
Gu and Foley 2007; Uga et al. 2007; Nonoue et al. 2008; Matsubara et al. 2008a; Maas et al. 2010).

The detection of QTLs for heading date has allowed further genetic analyses, such as the development of nearly isogenic lines (NILs), analysis of epistatic interactions among QTLs, and map-based cloning. Hdl, a major QTL for photoperiod sensitivity (daylength response), encodes a protein with zinc finger and CCT motifs and is an ortholog of CONSTANS, which is involved in long-day (LD) flowering promotion in Arabidopsis (Yano et al. 2000). Hd6 and $H d 3 a$, genes involved in photoperiod sensitivity, encode a casein kinase 2 alpha and an Arabidopsis FT-like protein, respectively (Takahashi et al. 2001; Kojima et al. 2002). Hd1 protein activates transcription of the rice florigen genes $H d 3 a$ and $R F T 1$ under short-day (SD) conditions while repressing transcription of these genes under LD conditions (Kojima et al. 2002; Tamaki et al. 2007; Komiya et al. 2008, 2009). Early heading date 1 (Ehdl), a promoter of heading, was detected on chromosome 10 by using a $\mathrm{BC}_{1} \mathrm{~F}_{1}$ population derived from a cross between cultivar T65 and an accession of another cultivated species, Oryza glaberrima; further analysis revealed that Ehdl encodes a B-type response regulator and activates $\mathrm{Hd} 3 \mathrm{a}$ and RFTl under SD conditions independently of $H d l$ (Doi et al. 2004). Recently, Xue et al. (2008) demonstrated that a major flowering repressor, $G h d 7$, encodes a protein with a CCT motif that represses transcription of Ehdl, Hd3a, and RFT1. These genetic and molecular studies have contributed substantially to our understanding of heading date in rice (Izawa 2007; Tsuji et al. 2008).

Although knowledge about the genetic control of rice heading has accumulated rapidly, the genetic mechanisms underlying the wide range of heading date variation still remain to be clarified. During the last decade, genetic analyses have been performed on a limited number of cross combinations to identify QTLs involved in heading date (Yonemaru et al. 2010). Furthermore, it has been very difficult to precisely compare QTLs detected in different studies due to the different levels of mapping resolution in each study (Yonemaru et al. 2010). In general, since QTLs are detected based on the allelic differences between parental lines, it is difficult to determine whether a particular QTL is shared among different cross combinations. Therefore, to understand genetic factors controlling heading date in diverse germplasm, a common parental line should be used for the development of mapping populations.

To comprehensively dissect naturally occurring variation in rice heading date, we performed a QTL analysis in 12 populations derived from crosses of the japonica cultivar Koshihikari, as a common parental line, with diverse cultivars that originate from various regions in Asia. Some of the QTLs detected in these populations were shared among several cross combinations, and their chromosomal locations corresponded to those of QTLs reported in other studies. In addition, we performed sequence analysis of several heading date genes that had been previously cloned by a map-based strategy, to examine the basis of the variation detected as QTLs in these populations. Based on the data from the QTL analysis and the sequence variation in the cloned heading date genes, we concluded that a large portion of the wide range of phenotypic variation for heading date in Asian cultivars could be generated by combinations of alleles with loss or gain of function in those QTLs.

\section{Materials and methods}

Plant materials

Twelve Asian rice cultivars were selected based on their geographical origins and cultivar groups (Kojima et al. 2005) (Table 1). We made crosses between those cultivars and a japonica cultivar, Koshihikari, as a common parental line to produce $F_{1} s$. Those $F_{1} s$ were self-pollinated to produce $\mathrm{F}_{2}$ progeny. For each population used in QTL mapping, $94 \mathrm{~F}_{2}$ plants and the two parental lines ( 24 plants of each) were raised in a paddy field at the National Institute of Agrobiological Sciences (NIAS) in Tsukuba, Japan, from April to November. The mean daylength during the cultivation period was $13.1 \mathrm{~h}$ in mid-April, $14.1 \mathrm{~h}$ in May, $14.6 \mathrm{~h}$ in June, $14.4 \mathrm{~h}$ in July, $13.5 \mathrm{~h}$ in August, and $12.4 \mathrm{~h}$ in September. Average temperature during the cultivation period was $17^{\circ} \mathrm{C}$ in May, $21^{\circ} \mathrm{C}$ in June, $24^{\circ} \mathrm{C}$ in July, $26^{\circ} \mathrm{C}$ in August, and $22^{\circ} \mathrm{C}$ in September. Cultivation management followed the standard procedures used at NIAS.

\section{Scoring of days-to-heading (DTH)}

We recorded the DTH of each $\mathrm{F}_{2}$ plant as the number of days from seeding to the appearance of the first panicle. For the parental cultivars, DTH was scored in 10 plants per line and mean values were calculated for each line.

DNA marker analysis

Total DNA of individual $\mathrm{F}_{2}$ plants and parental lines was extracted from leaves by the CTAB method (Murray and Thompson 1980). Two types of DNA markers, simple sequence repeat (SSR) and single-nucleotide polymorphism (SNP) markers, were used for linkage map construction. For polymerase chain reaction (PCR) of the SSR markers, we used a $10-\mu$ l reaction volume containing $0.5 \mu \mathrm{l}$ template DNA $\left(20 \mathrm{ng} \mu \mathrm{l}^{-1}\right), 5 \mu \mathrm{l} 2 \times$ Go-Taq Green 
Table 1 Geographical origin and heading date of rice accessions used in this study

\begin{tabular}{llllr}
\hline Accession $^{\text {a }}$ & Abbreviations & Origin & Cultivar group $^{\text {b }}$ & Days-to-heading $^{\text {c }}$ \\
\hline Koshihikari & KSH & Japan & A & $109 \pm 0.9$ \\
Qiu Zhao Zong & QZZ & China & C & $102 \pm 1.4$ \\
Tupa 121-3 & TUP & Bangladesh & B & 1.1 \\
Muha & MUH & India & B & $111 \pm 1.1$ \\
Davao1 & DAV & Philippines & C & $112 \pm 1.3$ \\
Toboshi & TOB & C & $115 \pm 2.0$ \\
Basilanon & BAS & B & $122 \pm 1.8$ \\
Deng Pao Zhai & DPZ & Philippines & C & $124 \pm 1.2$ \\
Khau Mac Kho & KMK & China & A & $125 \pm 2.6$ \\
Bei Khe & BKH & Vietnam & C & $127 \pm 1.6$ \\
Naba & NAB & Cambodia & C & $186 \pm 3.4$ \\
Khao Nam Jen & KNJ & India & A & $191 \pm 1.5$ \\
Bleiyo & BLE & Laos & C & \\
\hline
\end{tabular}

${ }^{a}$ All accessions were selected from world rice collections (WRC) (Kojima et al. 2005)

${ }^{\mathrm{b}}$ Cultivar group is based on the classification system of Kojima et al. (2005). Groups A, B, and C, correspond to japonica, Aus, and indica, respectively

${ }^{c}$ Days to heading was scored at the Experimental Field of National Institute of Agrobiological Sciences, Tsukuba, Ibaraki, Japan ( $\left.36^{\circ} \mathrm{N}\right)$. Days to heading is shown as mean $\pm \mathrm{SD}$

Master Mix (Promega, WI, USA) and $4.5 \mu \mathrm{l} \mathrm{H}_{2} \mathrm{O}$. Amplification was performed for 35 cycles $\left(1 \mathrm{~min}\right.$ at $94^{\circ} \mathrm{C}$, $1 \mathrm{~min}$ at $55^{\circ} \mathrm{C}$, and $2 \mathrm{~min}$ at $72^{\circ} \mathrm{C}$ ), followed by $7 \mathrm{~min}$ at $72^{\circ} \mathrm{C}$. The amplified products were separated by electrophoresis in a $3 \%$ agarose gel to detect polymorphisms. SSR markers used in the primary $\mathrm{F}_{2}$ analysis were selected from those reported by McCouch et al. (2002). The 409 SNPs used for genotyping were selected at a spacing of around $1,000 \mathrm{~kb}$ from genome wide SNP data (Ebana et al. 2010) (Suppl Table S1). The SNPs were detected using the BeadStation 500G system (illumina, San Diego, USA). All experimental procedures for the SNP typing followed the manufacturer's instructions. To obtain additional SSR markers showing polymorphism between Koshihikari and the other lines, we surveyed further genomic regions containing SSR motifs in the chromosomal regions of interest (Temnykh et al. 2000; McCouch et al. 2002; International Rice Genome Sequencing Project 2005). The resultant informative SSR markers were used for genotyping of $\mathrm{F}_{2}$ plants. Gene-specific markers for $H d l$ and $H d 6$ were also used in the QTL analysis (Suppl Table S2).

\section{Construction of linkage maps and QTL analyses}

QTL analysis of the $F_{2}$ populations was performed using genotype information based on the SSR and SNP markers. We used 86-164 SSR markers and 180-312 SNP markers distributed on the 12 rice chromosomes for the QTL analysis of each $\mathrm{F}_{2}$ population. Once a QTL was detected in a particular region of the genome, additional SSR markers were added to determine a more precise position and to increase the reliability of the estimated parameters for that putative QTL.

Linkage maps were constructed from the genotype data by using MAPMAKER/EXP 3.0 (Lander et al. 1987). The Kosambi map function was used to calculate genetic distances (Kosambi 1944). QTL analyses were performed using composite interval mapping (CIM) and interval mapping (IM) as implemented by the Zmapqtl program (model 6) provided in version 2.5 of Windows QTL Cartographer (Basten et al. 2005). Genome-wide threshold values $(\alpha=0.05)$ were used to detect putative QTLs based on the results of 1,000 permutations. The additive and dominant effects and phenotypic variance explained by each QTL were estimated at the peak LOD score.

\section{Sequence analysis}

All exon regions of the $H d 1, H d 3 a, R F T 1$, and $G h d 7$ genes were amplified by PCR from the genomic DNA of each rice variety with unique primers, using AmpliTaq polymerase (Applied Biosystems, Foster, CA, USA) or LA Taq polymerase (TaKaRa, Kyoto, Japan) and a PTC-100 PCR machine (MJ Research, Waltham, MA, USA). Amplification was performed by using 35 cycles at $94^{\circ} \mathrm{C}$ for $30 \mathrm{~s}$, $55^{\circ} \mathrm{C}$ for $30 \mathrm{~s}$, and $72^{\circ} \mathrm{C}$ for $1 \mathrm{~min}$, unless a different annealing temperature was determined (Suppl Table S3). In the case of $H d \sigma$, sequencing was conducted only for the third exon, in which a functional nucleotide polymorphism (FNP) was reported to exist within O. sativa (Yamane et al. 
2009). Amplified DNA fragments were purified and sequenced with the Sanger dideoxy terminator method on capillary sequencers (Applied Biosystems, Foster City, CA, USA). To ensure that the sequence data were of high quality (phred score $>30$ ), re-sequencing was performed when necessary. Each sequence read was individually mapped onto the Nipponbare reference coding region sequences to ensure that all exons of each gene were covered.

\section{Results}

Variations in heading date in $12 \mathrm{~F}_{2}$ populations

DTH of the 12 diverse cultivars and the common Koshihikari (KSH) parent varied from 91 (extremely early) to 191 (extremely late) under natural field conditions (Table 1). Among the accessions tested in this study, Qiu Zhao Zong (QZZ) showed the earliest heading. Four accessions, Deng Pao Zhai (DPZ), Khau Mac Kho (KMK), Bei Khe (BKH), and Naba (NAB), were relatively late heading, and two accessions, Khao Nam Jen (KNJ) and Bleiyo (BLE), showed extremely late heading (Table 1).

Frequency distributions of DTH for the $12 \mathrm{~F}_{2}$ populations are shown in Fig. 1. All 12 populations showed a wide range of DTH and a continuous distribution (Fig. 1). The segregation patterns were roughly classified into several categories. In the QZZ population, transgressive segregants for both early and late heading were observed (Fig. 1). In four $F_{2}$ populations (TUP, MUH, DAV, and TOB), a wide range of variation was observed even though the parents of each population had very similar DTH under field conditions. In particular, the TOB $F_{2}$ population showed an extreme amount of transgressive segregation toward late heading. In the BAS, DPZ, and KMK $\mathrm{F}_{2}$ populations, bimodal and transgressive segregation patterns were observed. In the BKH and NAB populations, both non-KSH parental lines showed relatively late heading, and extreme transgressive segregation toward late heading was observed in the $\mathrm{F}_{2}$ population. The most extreme transgressive segregation toward late heading was observed in the $\mathrm{F}_{2}$ population derived from BKH. In contrast, DTH of the two populations derived from parents with the longest DTH under field conditions (KNJ and BLE) were between those of the parental lines, and no transgressive segregation was observed.

QTLs involved in natural variation for heading date

QTLs detected in the $12 \mathrm{~F}_{2}$ populations are summarized in Table 2, Fig. 2, Suppl Figs. S1 and S2. Based on the CIM analysis, in the QZZ population, QTLs were detected on chromosome 7 (one near RM3670, another near RM5720), and chromosome 8 (RM5556); at each QTL, the KSH allele increased DTH. In the TUP population, QTLs were detected on chromosomes 2 (RM3789_1), 3 (RM5801), 6 ( $H d 1)$, and 8 (RM5432). The KSH allele increased DTH at the QTL on chromosome 6 and decreased DTH at the other three QTLs. In the MUH population, DTH variation could be explained by four QTLs: two on chromosome 3 (P1220 and P1710_1), one on chromosome 6 (RM3431), and one on chromosome 7 (P0388). The KSH alleles increased DTH at the QTL on chromosome 6 and at one of the two QTLs on chromosome 3 (P1220); at the other two QTLs, the KSH alleles decreased DTH. In the DAV population, variation for DTH could be explained by QTLs on chromosomes 6 (AH06000195 and P0013) and 8 (RM5556). The KSH allele decreased DTH at the QTL on chromosome 6 near AH06000195 and increased DTH at the other two QTLs. In the TOB population, we detected QTLs on chromosomes 6 (RM3805) and 8 (RM3374). The KSH allele at the QTL on chromosome 6 decreased DTH, whereas the KSH allele for the QTL on chromosome 8 increased DTH. In the BAS population, QTLs were mapped to chromosome 3 (Hd6) and chromosome 6 (RM6836_1). The KSH allele at the QTL on chromosome 3 decreased DTH, whereas the KSH allele at the QTL on chromosome 6 increased DTH. In the DPZ population, a single QTL was detected on chromosome 8 (RM5556); at this QTL, the KSH allele increased DTH. In the KMK population, QTLs were detected on chromosomes 2 (RM450), 3 (RM3867), and 6 (RM3431A). The KSH allele at the QTLs on chromosomes 2 and 3 decreased DTH; the KSH allele at the QTL on chromosome 6 increased DTH. In the BKH population, QTLs were detected on chromosomes 6 (RM3805) and 8 (RM5556). The KSH allele at the QTL on chromosome 6 decreased DTH; the KSH allele at the QTL on chromosome 8 increased DTH. In the NAB population, QTLs were detected on chromosomes 3 (RM6970), 6 (Hdl), 7 (RM5436), and 8 (RM3791); at these loci, only the KSH allele of the QTL on chromosome 6 increased DTH. In the KNJ population, QTLs were detected on chromosome 3 (RM6970) and chromosome 6 (RM3431A); at both QTLs, the KSH allele decreased DTH. In the BLE population, QTLs were detected on chromosome 2 (RM3316), chromosome 3 (RM6970), chromosome 6 (RM3805) and chromosome7 (RM1362_2); at all four QTLs, the KSH allele decreased DTH.

Of the 34 QTLs detected across the $12 \mathrm{~F}_{2}$ populations, 19 each explained more than $15 \%$ of the phenotypic variance in the populations where they were detected, indicating that those QTLs harbor genes with major effects. All 34 QTLs mapped within eight chromosomal regions (Fig. 2) corresponding to previously identified heading-date QTLs such 

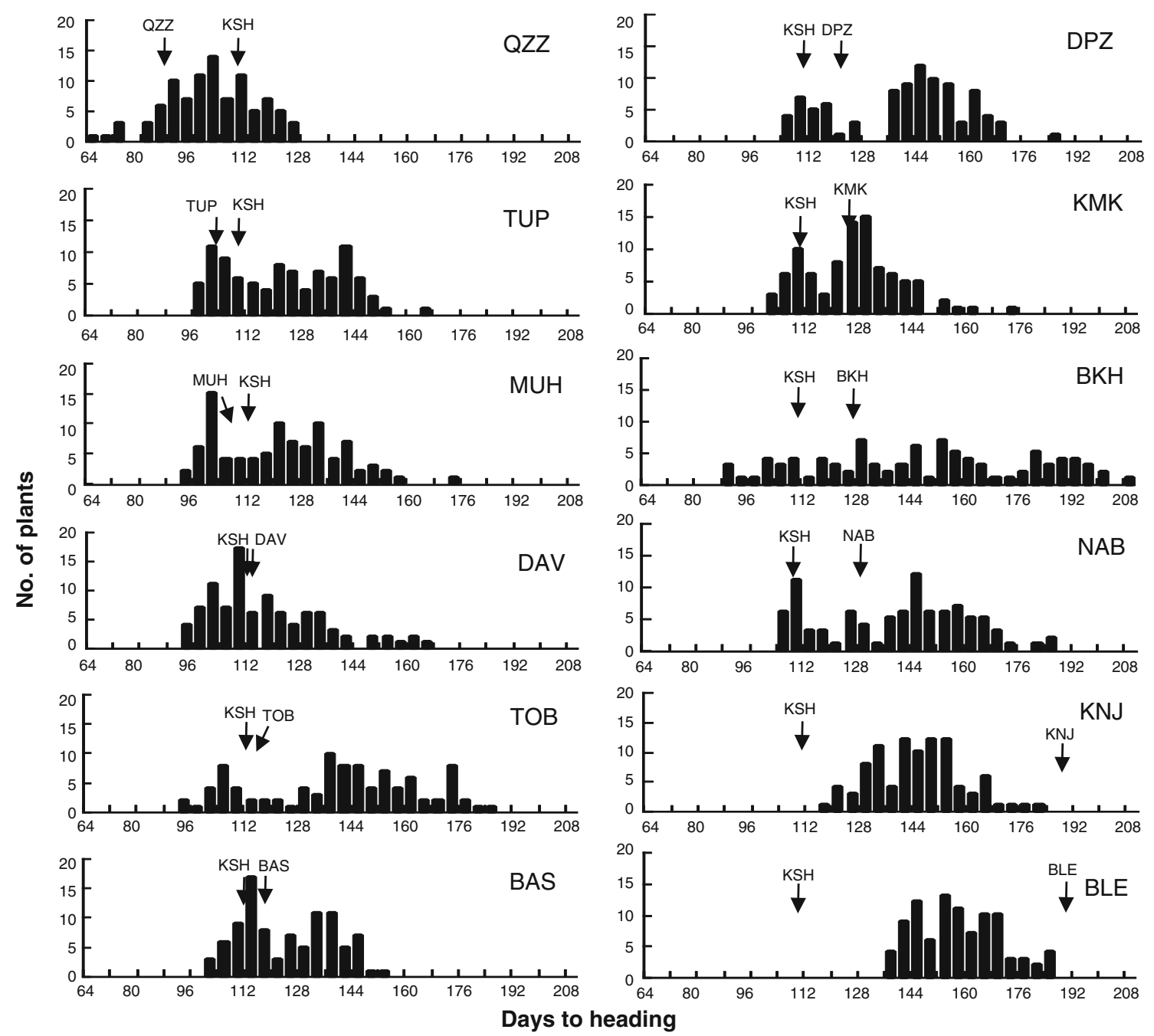

Fig. 1 Frequency distributions of days to heading (DTH) in $\mathrm{F}_{2}$ populations derived from crosses between Koshihikari and 12 diverse Asian cultivars. DTH was scored at the Experimental Field of

as $H d 1$ (Yano et al. 2001), Hd2 (Yamamoto et al. 1998), Hd3a/RFTl (Kojima et al. 2002), Ghd7 (Xue et al. 2008), Hd5 (Lin et al. 2003), Hd6/Hd16 (Takahashi et al. 2001; Matsubara et al. 2008a), Hd7 (Yamamoto et al. 2000), and Hd9 (Lin et al 2002) (Fig. 2; Table 2). It should be noted that three genomic regions (those containing $\mathrm{Hdl}, \mathrm{Hd}$, and Hd6/Hd16) were each associated with DTH variation in seven populations (Fig. 2).

To examine validity of QTL detected in the CIM analysis, we also performed IM analysis to know a potential risk of a false positive detection of the QTLs. Based on IM analysis, several QTL, which were detected in the CIM analysis, were not detected (Table 2, Suppl Fig. S2). Of 34 QTLs detected in CIM, 11 QTLs on chromosome 2, 3, 6, 7 and 8 were not detected in IM analysis. Among them, only one QTL showing a relatively a large phenotypic
National Institute of Agrobiological Sciences, Tsukuba, Ibaraki, Japan $\left(36^{\circ} \mathrm{N}\right)$. Arrows show the mean DTH in the parental lines. Parental lines are denoted by the abbreviations given in Table 1

contribution in the CIM analysis (more than 10\% PVE) was not detected in the IM analysis (Table 2);

Sequence analysis of genes for heading date

Our QTL analyses strongly suggested that several previously cloned genes for heading date could explain a large portion of the phenotypic variation in each $F_{2}$ population, indicating that there should be allelic differences for gene function at these loci. Therefore, to search for functional differences in these heading date genes, we sequenced the genomic regions of candidate genes $H d l, H d 6, H d 3 a, R F T 1$, and $G h d 7$, which were located near many of the QTLs detected in this study. $\mathrm{KSH}$ is presumed to have a functional allele for each of these genes since it contains the same sequences as are found in the Nipponbare genome (Nonoue et al. 2008). 
Table 2 Heading date QTLs detected in 12 F2 populations derived from crosses between Koshihikari and Asian rice cultivars

\begin{tabular}{|c|c|c|c|c|c|c|c|c|c|c|}
\hline Population & $\begin{array}{l}\text { Marker } \\
\text { type }\end{array}$ & Chr & $\begin{array}{l}\text { Nearest } \\
\text { marker }\end{array}$ & $\begin{array}{l}\text { Physical position } \\
\text { of QTL (Mbp) }\end{array}$ & $\mathrm{LOD}^{\mathrm{a}}$ & $\begin{array}{l}\text { Additive } \\
\text { effect }^{\mathrm{b}}\end{array}$ & $\begin{array}{l}\text { Dominance } \\
\text { effect }^{\mathrm{c}}\end{array}$ & $\begin{array}{l}\text { PVE } \\
(\%)^{d}\end{array}$ & $\mathrm{QTL}^{\mathrm{e}}$ & $\mathrm{IM}^{\mathrm{f}}$ \\
\hline \multirow[t]{3}{*}{ QZZ } & \multirow[t]{3}{*}{ SSR } & 7 & RM3670 & 14.94 & 19.40 & 9.73 & 9.42 & 43.32 & Ghd7 & \\
\hline & & 7 & RM5720 & 29.33 & 4.19 & 5.66 & 1.56 & 8.53 & $H d 2$ & n.d. \\
\hline & & 8 & RM5556 & 4.59 & 8.82 & 8.24 & 4.18 & 19.29 & $H d 5$ & \\
\hline \multirow[t]{4}{*}{ TUP } & \multirow[t]{4}{*}{ SSR } & 2 & RM3789_1 & 34.77 & 4.35 & -4.12 & -0.33 & 2.95 & $H d 7$ & n.d. \\
\hline & & 3 & RM5801 & 33.57 & 11.85 & -7.83 & 1.02 & 9.40 & $H d 6 / H d 16$ & n.d. \\
\hline & & 6 & Hd1 & 9.96 & 41.65 & 19.37 & 4.52 & 70.21 & $H d 1$ & \\
\hline & & 8 & RM5432 & 4.07 & 7.67 & -6.10 & 0.72 & 5.89 & $H d 5$ & n.d. \\
\hline \multirow[t]{4}{*}{ MUH } & \multirow[t]{4}{*}{ SNP } & 3 & P1220 & 2.08 & 4.28 & 2.41 & 7.78 & 4.54 & $H d 9$ & n.d. \\
\hline & & 3 & P1710_1 & 34.28 & 6.72 & -7.30 & 1.32 & 9.33 & $H d 6 / H d 16$ & \\
\hline & & 6 & RM3431 & 8.64 & 28.55 & 19.06 & 7.01 & 63.87 & $H d 1$ & \\
\hline & & 7 & P0388 & 28.22 & 4.12 & -4.60 & 5.72 & 4.79 & $H d 2$ & n.d. \\
\hline \multirow[t]{3}{*}{ DAV } & \multirow[t]{3}{*}{ SNP } & 6 & AH06000195 & 3.05 & 6.34 & -9.00 & -6.32 & 16.98 & $H d 3 a / R F T 1$ & n.d. \\
\hline & & 6 & P0013 & 8.32 & 8.75 & 9.39 & 5.99 & 19.06 & $H d l$ & \\
\hline & & 8 & RM5556 & 4.59 & 12.12 & 12.12 & 5.54 & 28.96 & $H d 5$ & \\
\hline \multirow[t]{2}{*}{ TOB } & \multirow[t]{2}{*}{ SNP } & 6 & RM3805 & 2.79 & 8.21 & -10.69 & -7.68 & 14.29 & $H d 3 a / R F T 1$ & \\
\hline & & 8 & RM3374 & 3.75 & 28.83 & 23.24 & 12.97 & 59.88 & $H d 5$ & \\
\hline \multirow[t]{2}{*}{ BAS } & \multirow[t]{2}{*}{ SSR } & 3 & Hd6 & 32.37 & 8.38 & -5.14 & 7.21 & 12.59 & Hd6/Hd16 & n.d. \\
\hline & & 6 & RM6836-1 & 9.31 & 24.71 & 14.67 & 4.40 & 58.33 & $H d 1$ & \\
\hline DPZ & SNP & 8 & RM5556 & 4.59 & 17.12 & 22.18 & 17.25 & 53.62 & $H d 5$ & \\
\hline \multirow[t]{3}{*}{ KMK } & \multirow[t]{3}{*}{ SSR } & 2 & RM450 & 30.61 & 6.87 & -6.92 & -1.69 & 9.31 & $H d 7$ & n.d. \\
\hline & & 3 & RM3867 & 32.79 & 14.02 & -9.85 & 0.21 & 22.36 & $H d 6 / H d 16$ & \\
\hline & & 6 & RM3431A & 8.72 & 17.94 & 12.34 & -0.54 & 33.14 & $H d 1$ & \\
\hline \multirow[t]{2}{*}{ BKH } & \multirow[t]{2}{*}{ SSR } & 6 & RM3805 & 2.85 & 7.54 & -13.59 & -13.06 & 11.64 & $H d 3 a / R F T 1$ & \\
\hline & & 8 & RM5556 & 5.41 & 23.45 & 23.51 & 21.37 & 42.01 & $H d 5$ & \\
\hline \multirow[t]{4}{*}{ NAB } & \multirow[t]{4}{*}{ SSR } & 3 & RM6970 & 33.60 & 8.49 & -9.15 & 3.78 & 9.52 & Hd6/Hd16 & \\
\hline & & 6 & $\mathrm{Hd} 1$ & 9.33 & 30.90 & 19.93 & 10.90 & 43.25 & $H d 1$ & \\
\hline & & 7 & RM5436 & 9.11 & 6.24 & -7.90 & 4.50 & 6.13 & Ghd7 & n.d. \\
\hline & & 8 & RM3791 & 4.52 & 9.48 & -9.01 & 4.06 & 10.32 & $H d 5$ & \\
\hline \multirow[t]{2}{*}{ KNJ } & \multirow[t]{2}{*}{ SSR } & 3 & RM6970 & 33.71 & 21.37 & -13.16 & 4.44 & 54.11 & $H d 6 / H d 16$ & \\
\hline & & 6 & RM3431A & 8.74 & 12.36 & -8.90 & 4.31 & 22.18 & $H d 1$ & \\
\hline \multirow[t]{4}{*}{ BLE } & \multirow[t]{4}{*}{ SSR } & 2 & RM3316 & 32.31 & 4.55 & -5.49 & -0.41 & 9.56 & $H d 7$ & n.d. \\
\hline & & 3 & RM6970 & 32.99 & 5.51 & -4.87 & 4.50 & 9.70 & Hd6/Hd16 & \\
\hline & & 6 & RM3805 & 3.19 & 14.53 & -9.49 & -6.11 & 28.13 & $H d 3 a / R F T 1$ & \\
\hline & & 7 & RM1362_2 & 29.91 & 9.34 & -7.88 & -1.01 & 18.23 & $H d 2$ & \\
\hline
\end{tabular}

All genetic parameters were calculated by Composit Interval mapping function (CIM) in QTL Cartographer ver. 2.5 (Basten et al. 2005) SSR simple sequence repeat, SNP single-nucleotide polymorphism

${ }^{a}$ Log-likelihood value

b Additive effect of Koshihikari allele on days-to-heading

c Dominance effect of Koshihikari allele

${ }^{\mathrm{d}}$ Percent of phenotypic variance explained by QTL. LOD threshold to detect QTLs was determined in each F2 population (Figure S1)

e Previously identified QTL(s) corresponding with the QTLs detected in this study based on their physical positions

${ }^{\mathrm{f}}$ Results of Interval Maping (IM) analysis. QTLs not detected in IM analysis are indicated by n.d.

Within $H d l$, a number of mutations were observed that would be expected to cause amino acid substitutions in the Hd1 protein. In addition, single-nucleotide polymorphisms (SNP) or indels (insertion/deletion mutations) that generated a premature stop codon in the exon region were observed in DAV, BAS, KMK, MUH, NAB, TUP, and QZZ (Fig. 3). These mutations would result in a full or partial deletion of the NLS domain at the C-terminus of the 


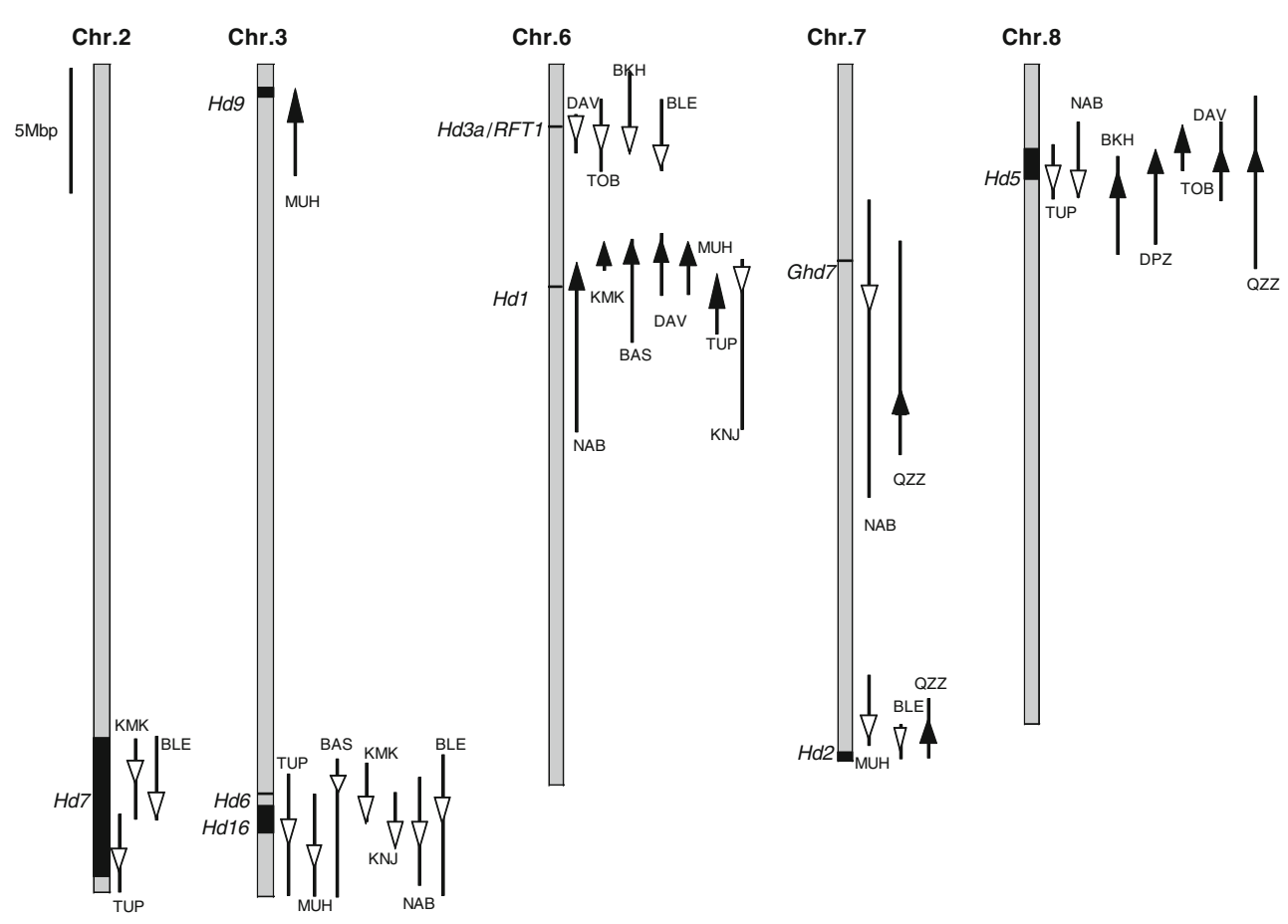

Fig. 2 Chromosomal locations of QTLs detected in $12 F_{2}$ populations. Most likely QTL positions detected in each population (Table 2) are shown by arrowheads; confidence intervals (2-LOD reduction on each side) are indicated by bars extending from the arrowheads. Cultivar abbreviations are as shown in Table 1. The direction of additive effect of the Koshihikari (KSH) allele at each QTL detected is shown by a white (earlier heading) or black (later

Hd1 protein. The most extreme case was found in QZZ, in which the amino acid sequences within the zinc finger motif were changed due to a frameshift caused by an indel. Based on these results, these seven accessions appear to contain alleles with a loss of function at $H d l$.

In the predicted amino acid sequence of $\mathrm{Hd} 3 \mathrm{a}$, only one amino acid substitution (near the C-terminus) could be detected in TOB, NAB, DVA, DPZ, MUH, TUP, and BLE, as compared with the KSH allele (data not shown). In the RFT1 gene, on the other hand, a number of SNPs corresponding to amino acid substitutions were observed in KMK, DAV, TOB, BKH, NAB, DPZ, MUH, and TUP (Fig. 3). It should also be noted that DAV, TOB, BKH, BLE appear to have one allele; and NAB, DPZ, MUH, and TUP appear to have another (Fig. 3).

In Ghd7, several SNPs corresponding to amino acid substitutions were found in BLE, TOB, BKH, NAB, DAV, and DPZ, as compared with the KSH allele (Fig. 3); all of these except for DPZ had the same predicted amino acid sequence. In QZZ, no PCR fragments could be amplified from any $G h d 7$ exon region. Based on PCR examination of the regions flanking $G h d 7$ by using QZZ BAC clones covering the corresponding genomic region, it appeared that the genomic segment $(\sim 40 \mathrm{~kb})$ normally harboring heading) arrowhead. Physical positions of Hd6 (Takahashi et al. 2001), Hdl (Yano et al. 2000), Hd3a (Kojima et al. 2002), RFT1 (Kojima et al. 2002), and Ghd7 (Xue et al. 2008) are shown by horizontal bars. The positions of $H d 7$ (Yamamoto et al. 2000), $H d 9$ (Lin et al. 2002), Hd16 (Matsubara et al. 2008a), Hd2 (Yamamoto et al. 1998, 2000) and $H d 5$ (Lin et al. 2003) are shown as boxes defined by the positions of flanking markers

Ghd7 was completely deleted, as reported previously for the Chinese rice variety Zhenshan 97 (Xue et al. 2008).

For $H d 6$, it has been previously reported that the coding sequence is highly conserved and that natural mutations have been suppressed during its evolution in the genus Oryza (Yamane et al. 2009). A non-synonymous base substitution generating a premature stop codon was found in a limited number of Japanese japonica varieties, including KSH (Takahashi et al. 2001; Matsubara et al. 2008a; Yamane et al. 2009). Sequencing a 500-bp genomic region including the non-synonymous substitution site in the 12 other cultivars used in this study demonstrated that this functional nucleotide polymorphism (FNP) was not present in any of the lines (data not shown), suggesting that all of the cultivars except for KSH carry a functional allele at the $H d 6$ locus.

\section{Discussion}

Heading date in rice has been intensively analyzed over the last two decades. These efforts have contributed to our understanding of the genetic and molecular control of this trait (Izawa 2007; Tsuji et al. 2008). In particular, cloning 


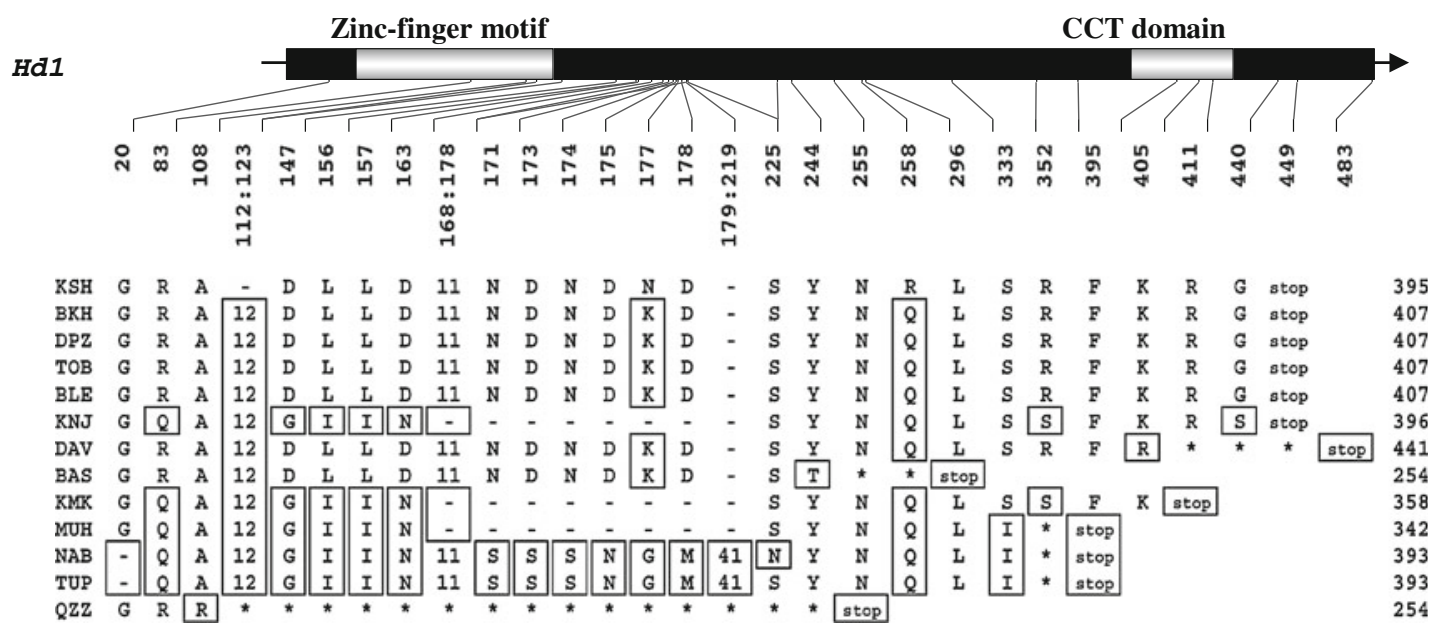

Phosphatidylethanolamine -

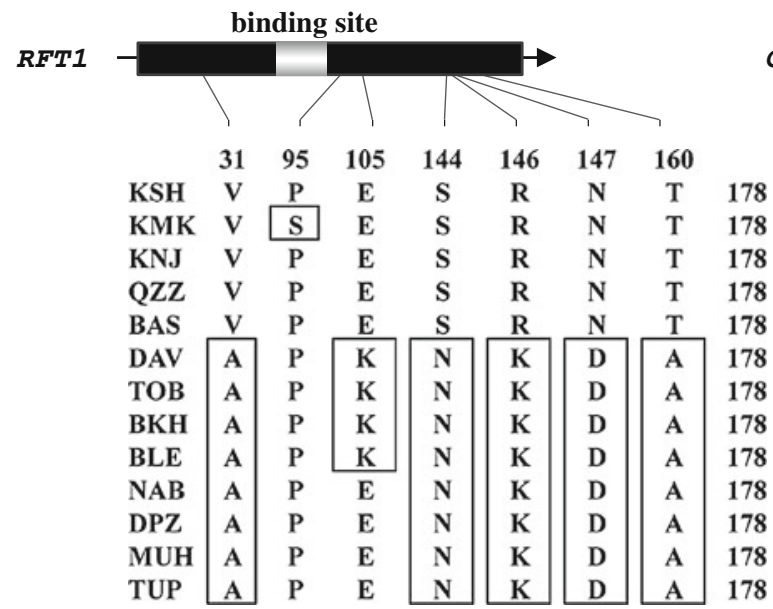

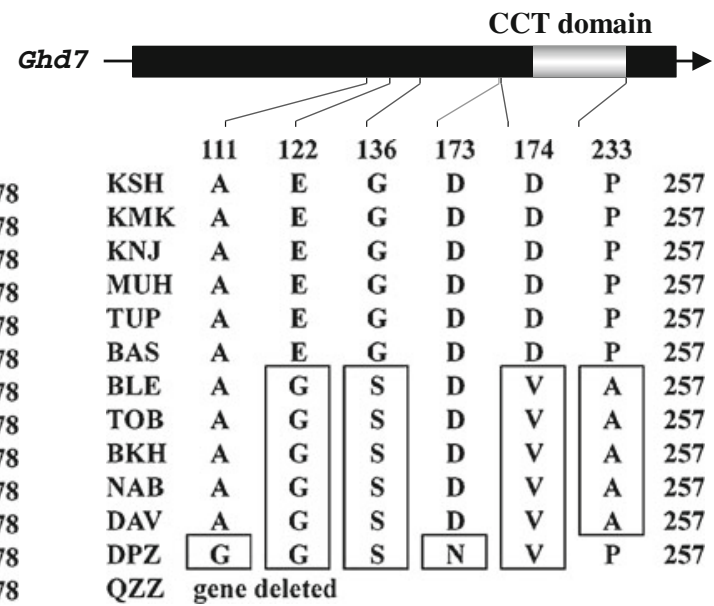

Fig. 3 Substitutions (boxed) and indels (-) of amino acids observed in heading date genes $H d l, R F T 1$, and Ghd7. The known domains within each gene are indicated as light-colored regions with labels above them. Numbers under the gene bars indicate sites of sequence changes; numbers on the right show the total length of each predicted amino acid sequence. The regions with amino acid changes due to frameshifts are labeled with an asterisk. Stop stop codon

compared them with the common japonica variety KSH. These 12 cultivars exhibited a wide range of DTH under natural field conditions at Tsukuba, Japan (Table 1). QTL analyses using $F_{2}$ populations from 12 different crosses (KSH by each of the 12 diverse cultivars) revealed that one to four QTLs with major effects were involved in natural variation in heading date in each population. The chromosomal locations of these major genetic factors corresponded well with previously identified QTLs: $H d 1, H d 2$, Hd3a/RFT1, Ghd7, Hd5, Hd6/Hd16, Hd7, and Hd9. Although it could not be ruled out that other tightly linked genes controlling DTH exist in these chromosomal regions, we suggest that the majority of natural variation in flowering time is generated by the actions of multiple alleles at the QTLs detected in this study. Furthermore, we suggest that at least some of the QTLs detected in this study are identical to the previously identified QTLs. 
More than $65 \%$ of the total phenotypic variance in 10 of the 12 populations could be explained by the QTLs detected in this study. However, less than $60 \%$ of the variance in $\mathrm{F}_{2}$ populations derived from DPZ and BKH could be explained by the QTLs detected in this study. In particular, although only one QTL was detected in the DPZ population, clear transgressive segregation for late heading was observed. This transgressive segregation could not be explained by the effect of a single QTL, indicating that additional QTLs must be involved in the variation in heading date in the DPZ population.

Transgressive segregation was observed in most of populations; the exceptions were the populations derived from crosses with the latest-heading cultivars, KNJ and BLE. The transgressive segregation could be explained by the combination of $\mathrm{KSH}$ and other parental alleles having both positive and negative effects on DTH. In fact, both directions of effect were detected in the $10 \mathrm{~F}_{2}$ populations that showed transgressive segregation. In the KNJ and BLE $\mathrm{F}_{2}$ populations, a decrease in DTH was associated with the $\mathrm{KSH}$ allele for all QTLs detected in those populations. On the other hand, although an increase in DTH was associated with the KSH alleles of all three QTLs detected in QZZ, transgressive segregation was observed toward both late and early heading. This segregation pattern could not be clearly explained by the QTLs detected in the QZZ $F_{2}$ population.

In two $\mathrm{F}_{2}$ populations (TOB and $\mathrm{BKH}$ ), the same patterns of allelic differences were detected at Hd3a/RFT1 and Hd5: the TOB and BKH parents both contributed lateheading alleles at $H d 3 a / R F T 1$ and early-heading alleles at $H d 5$. These two populations showed a very wide range of DTH, and extremely late transgressive segregants were observed. However, variation in DTH was different between the two populations: BKH showed late heading compared with that of TOB, and the distribution of DTH was shifted toward late heading in the $\mathrm{BKH}$ population compared with that of TOB. This observation may imply that additional unknown late-heading factors in $\mathrm{BKH}$ or early-heading alleles in TOB might be involved in the variation observed in each population.

In the DAV and TOB $F_{2}$ populations, two of the same QTLs, Hd3a/RFT1 and Hd5, were detected. The additive effects of RFT1 were almost same between DAV and TOB, and both accessions harbored an early allele at $H d 5$, although different magnitudes of additive effects for $H d 5$ were observed. The main difference detected between the cultivars was that the DAV population contained an earlyheading allele of $\mathrm{Hdl}$ (contributed by DAV), whereas no QTL was detected near $H d l$ in the TOB population.

In a comparison between $\mathrm{KNJ}$ and BLE, both $\mathrm{F}_{2}$ populations showed a similar pattern of non-transgressive segregation. However, the only QTL in common between the populations was that detected near $H d 6 / H d 16$. These results indicated that the strong daylength response in each cultivar was controlled by a different combination of alleles at QTLs for heading date.

For QZZ, extremely early-heading plants were observed in the $F_{2}$ population. This segregation pattern was very similar to that of an $\mathrm{F}_{2}$ population derived from a cross between Hayamasari and Kasalath (Nonoue et al. 2008). The same two QTLs involved in early heading were detected in both analyses. It has also been reported that allelic variation in the $H d 2$ region on the distal end of the long arm of chromosome 7 could be involved in extremely early heading in rice (Fujino and Sekiguchi 2005; Gu and Foley 2007). In our study, a QTL near $H d 2$ was detected in the QZZ population, with the QZZ allele contributing to early heading (Table 2; Fig. 2). Therefore, we conclude that $G h d 7, H d 2$, and $H d 5$ play important roles in the genetics of extremely early heading.

The Hd6 QTL is located in the distal end of the long arm of chromosome 3 . KSH possesses a loss-of-function allele at Hd6 (Matsubara et al. 2008a). Recently, a new QTL, Hd16, was also detected near $H d 6$. In this region, it was difficult to distinguish between $\mathrm{Hd} 6$ and $\mathrm{Hd} 16$ due to their close linkage. However, two LOD peaks were detected in BAS and KNJ, suggesting that both loci might contribute to the variation for heading date. This assumption will need to be tested by performing high-resolution mapping of this region.

In QTL analysis, QTLs are detected based on the effects of different alleles on the target traits being measured. At each QTL detected in this study, we could compare the effects of alleles from different cultivars by using the allele of $\mathrm{KSH}$, a common parent, as a standard. For example, $\mathrm{KSH}$ has a functional allele of $\mathrm{Hdl}$ that is the same as that found in Nipponbare, with a 36-bp deletion in the exon (Yano et al. 2000; Nonoue et al 2008). Depending on whether the effect of the KSH allele was positive or negative in a given population, one could predict whether the Hdl allele in the other parent would have reduced or elevated function, respectively. Based on this assumption, we speculated that TUP, MUH, DAV, BAS, KMK, and NAB would possess a reduced-function or loss-of-function allele at $H d l$, and that KNJ would possess an $H d l$ allele with an enhanced level of function. Sequence analysis of $H d l$ strongly supports the above assumption. A premature stop codon was detected in the Hdl alleles of TUP, MUH, DAV, BAS, KMK, and NAB, indicating that these are very likely to be loss-of-function alleles. On the other hand, it is difficult to explain how the amino acid changes seen in the KNJ allele would lead to a gain-of-function compared with the $\mathrm{KSH}$ allele.

Xue et al. (2008) identified a repressor of heading, Ghd7: loss of Ghd7 function results in early heading. In QZZ, we detected a large deletion that appeared to include 
the entire $G h d 7$ sequence. The allelic effect of the KSH allele at the QTL near Ghd7 was positive in the QTL analysis of the QZZ $F_{2}$ population (Table 2), consistent with loss of function of $G h d 7$ in QZZ.

Recently, Hagiwara et al. (2009) reported that an amino acid change $(\mathrm{E} 105 \mathrm{~K})$ in the coding region of RFTl was involved in a significant loss of function in some Asian cultivated rice and its wild relatives, resulting in late heading. In the present study, QTLs with a large effect were detected in the region of $H d 3 a / R F T 1$ in four populations (DAV, TOB, BKH, and BLE). In each case, the $\mathrm{KSH}$ allele decreased DTH, suggesting that the function of RFT1 in the four diverse parents might have been decreased or lost. We could not detect any clear polymorphism affecting the function of $\mathrm{Hd} 3 \mathrm{a}$ (data not shown), whereas functional polymorphism (loss-of-function mutation $\mathrm{E} 105 \mathrm{~K}$ ) was detected in the predicted RFT1 protein sequence in DAV, TOB, BKH, and BLE. These results clearly suggest that $R F T 1$ may have a significant role in generating natural variation for heading date.

For $H d 2$ and $H d 5$, additive effects of the KSH alleles were seen in both directions (i.e., toward either increased or decreased DTH), depending on the $\mathrm{F}_{2}$ population. As mentioned above, this range of additive effects must reflect the functional status of gene(s) located within the QTLs. Unfortunately, neither $H d 2$ nor $H d 5$ have been cloned yet. The functional status of these genes will need to be tested by the cloning and sequencing of these two important QTLs for heading date.

Based on a previous sequence analysis, $\mathrm{KSH}$ has a nonfunctional allele at $H d 6$, which can be identified by a SNP (Matsubara et al. 2008a). In this study, it was revealed that none of the 12 other cultivars had this SNP at $H d 6$ (data not shown), suggesting that all 12 cultivars may have a functional allele at $H d 6$. Based on this sequence polymorphism, we expected to detect a QTL near Hd6 in all of the populations. However, no QTL was detected in the $H d 6$ region of QZZ, DAV, TOB, DPZ, or BKH (Fig. 2; Table 2). It is interesting to note that a $\mathrm{KSH}$ allele at $H d 5$ with a large positive effect on DTH was detected in all populations in which no QTL was detected in the Hd6 region. This indicates that genetic interaction between $H d 6 / H d l 6$ and $H d 5$ is likely to be involved in the expression of heading date. Alternatively, segregation of a large phenotypic effect of Hd5 may mask the action of $H d 6$.

Temperature and rainfall are major determinants of rice growing season, and DTH is important characteristic of rice cultivars. Natural or artificial selection of DTH has affected not only specific genes but also combinations of genes/QTLs. On the other hand, rice is an autogamous (self-fertilizing) crop in which natural crossing events among cultivars are extremely rare. Allele combinations at different QTLs that determine DTH and daylength response are expected to be characteristic of specific cultivar groups. The 12 cultivars used in this study have been classified into three cultivar groups, A, B, and C, which correspond to japonica, Aus, and indica, respectively (Table 1) (Kojima et al. 2005). The japonica cultivars studied here were KMK, KNJ, and KSH. The difference in DTH of KMK and KNJ is largely explained by the effect of the QTL near $H d l$, with an early allele in KMK and a late allele in $\mathrm{KNJ}$, and may reflect the needs of their respective growing environments. KMK is grown in mountainous areas of Vietnam, which may require photoperiod insensitive rice adapted to a relatively short growth period. On the other hand, KNJ seems to be grown in plains areas of tropical regions and a long growth period allows to raise high photoperiod sensitive rice. Three cultivars (TUP, MUH, and BAS) classified into group B by Kojima et al. (2005), exhibited relatively early heading among the cultivars in this study. This result is consistent with the typical Aus characteristics of photoperiod insensitivity and short growing period. The $F_{2}$ populations derived from the three group B cultivars showed similar segregation patterns for DTH; the QTL and sequence analyses suggested that these patterns were mainly determined by allelic differences in $H d 1$ and $H d 6 / H d 16$. On the other hand, although the seven cultivars classified into group C (QZZ, DAV, TOB, DPZ, $\mathrm{BKH}, \mathrm{NAB}, \mathrm{KMK}, \mathrm{KNJ}$, and BLE) showed a wide range of DTH under the natural field conditions (Table 1), less polymorphism was detected in $H d l$ and $G h d 7$ in group C (indica) than within groups A or B. In addition, based on the QTL analysis, allelic differences in RFT1 and $H d 5$ were more likely to be involved in generation of natural variation in DTH among cultivars in group $\mathrm{C}$ than among the other two groups. Garris et al. (2005) suggested that cultivar groups Aus and Aman, other cultivar's group in indica, had narrow and wide genetic diversity, respectively, based on SSR analysis. A similar result was observed in cultivar groups $\mathrm{B}$ and $\mathrm{C}$ (which correspond to Aus and Aman) based on their RFLPs (Kojima et al. 2005). Based on results in this study, it is likely that there is a relationship between allele combinations for the QTLs and cultivar groups or geographical distribution. However, the limited number of accessions in this study representing each cultivar group and geographical origin did not allow us to draw a clear conclusion. Future studies with more rice accessions will be needed to allow us to clearly establish the relationships between cultivar groups and genetic variation in QTLs for heading date.

Takahashi et al. (2009) demonstrated that variations in Hd1 proteins, $H d 3$ a promoters, and Ehdl expression levels largely contributed to the diversity of flowering time in cultivated rice. In this study, we confirmed that $H d l$ could be one of the major factors determining heading date in Asian cultivars; moreover, RFT1, rather than $H d 3 a$, contributed to 
heading date diversity in the rice cultivars included in this study. Furthermore, Ghd7, Hd5, and Hd6/Hd16 appeared to be important genetic factors for the determination of heading date in these rice cultivars. It should be noted that we did not detect any QTLs in the region of Ehdl (chromosome 10) in this study. This finding implies that, despite its important role for the promotion of flowering (Doi et al. 2004; Xue et al. 2008, Matsubara et al. 2008b; Park et al. 2008), the Ehdl gene does not contribute very much to heading date diversity in rice. Presumably, several of the QTLs detected in this study, such as $G h d 7, H d 2$ and $H d 5$, might modulate the expression of $E h d l$, and consequently modify expression of florigen genes Hd3a and RFTl (Xue et al. 2008).

Taking together the allelic differences found in the QTL analyses and the sequence variation at genes for heading date, our data strongly suggest that a large portion of the wide range of phenotypic variation in heading date and daylength response could be generated by combinations of different alleles, possibly both loss and gain of function, of QTLs Hdl, Hd2, RFT1, Ghd7, Hd5, and Hd6/Hd16. In general, it is difficult to detect all QTLs, particularly QTLs with minor effects, in primary mapping populations such as $\mathrm{F}_{2}$ populations (Ebitani et al. 2005; Uga et al. 2007; Takai et al. 2007; Ando et al. 2008). Although we successfully detected several major QTLs by using $F_{2}$ populations, it is very likely that some QTLs with minor effects might be also involved in the phenotypic variation seen in these $F_{2}$ populations. On the other hand, we also need to verify the effect of QTLs with relatively minor effects, which were not detected in the IM analysis. Therefore, it will be necessary to search again for heading date QTLs by using advanced progeny. In addition, DTH in the $\mathrm{F}_{2}$ plants was scored only under a single environment, natural field conditions at Tsukuba, Japan. It is possible that additional novel QTLs might be involved in differences in DTH under diverse environmental conditions, such as temperature and daylength, including artificial SD and LD conditions. Currently, we are developing populations of chromosome segment substitution lines (CSSLs) for 10 of the accessions (Fukuoka et al. 2010). It would be interesting to discover additional QTLs with minor or epistatic effects under diverse environmental conditions using these CSSLs, resulting in understanding more comprehensively the genetic factors that induce natural variation in heading date of Asian cultivated rice.

Acknowledgments We thank to the Technical Support Section of NIAS for the management of rice field. This work was supported by grants from the Ministry of Agriculture, Forestry and Fisheries of Japan (Integrated Research Project for Plant, Insect and Animal using Genome Technology QT-1005 and Genomics for Agricultural Innovation NVR-0001 to M. Yano, and Genomics for Agricultural Innovation QTL-5003 to J. Wu).
Open Access This article is distributed under the terms of the Creative Commons Attribution Noncommercial License which permits any noncommercial use, distribution, and reproduction in any medium, provided the original author(s) and source are credited.

\section{References}

Ando T, Yamamoto T, Shimizu T, Ma XF, Shomura A et al (2008) Genetic dissection and pyramiding of quantitative traits for panicle architecture by using chromosomal segment substitution lines in rice. Theor Appl Genet 116:881-890

Basten CJ, Weir BS, Zeng ZB (2005) QTL Cartographer, version 1.17. Department of Statistics, North Carolina State University, Raleigh, p 189

Doi K, Izawa T, Fuse T, Yamanouchi U, Kubo T et al (2004) Ehdl, a B-type response regulator in rice, confers short-day promotion of flowering and controls FT-like gene expression independently of Hd1. Genes Dev 18:926-936

Ebana K, Yonemaru J, Fukuoka S, Iwata H, Kanamori H, Namiki N, Nagasaki H, Masahiro Y (2010) Genetic structure revealed by a whole-genome single-nucleotide polymorphism survey of diverse accessions of cultivated Asian rice (Oryza sativa L.). Breed Sci 60:390-397

Ebitani T, Takeuchi Y, Nonoue Y, Yamamoto T, Takeuchi Y et al (2005) Construction and evaluation of chromosome segment substitution lines carrying overlapping chromosome segments of indica rice cultivar 'Kasalath' in a genetic background of japonica elite cultivar 'Koshihikari'. Breed Sci 55:65-73

Fujino K, Sekiguchi H (2005) Identification of QTLs conferring genetic variation for heading date among rice varieties at the northern-limit of rice cultivation. Breed Sci 55:141-146

Fukuoka S, Nonoue Y, Yano M (2010) Germplasm enhancement by developing advanced plant materials from diverse rice accessions. Breed Sci 60:509-517

Garris AJ, Tai TH, Coburn J, Kresovich S, McCouch SR (2005) Genetic structure and diversity in Oryza sativa L. Genetics 169:1631-1638

Gu XY, Foley ME (2007) Epistatic interactions of three loci regulate flowering time under short and long daylengths in a backcross population of rice. Theor Appl Genet 114:745-754

Hagiwara WE, Uwatoko N, Sasaki A, Matsubara K, Nagano H et al (2009) Diversification in flowering time due to tandem FT-like gene duplication, generating novel Mendelian factors in wild and cultivated rice. Mol Ecol 18:1537-1549

Hayama R, Coupland G (2004) The molecular basis of diversity in the photoperiodic flowering responses of Arabidopsis and rice. Plant Physiol 135:677-684

Hittalmani S, Huang N, Courtois B, Venuprasad R, Shashidhar HE et al (2003) Identification of QTL for growth- and grain yieldrelated traits in rice across nine locations of Asia. Theor Appl Genet 107:679-690

International Rice Genome Sequencing Project (2005) The map-based sequence of the rice genome. Nature 436:793-800

Izawa T (2007) Adaptation of flowering-time by natural and artificial selection in Arabidopsis and rice. J Exp Bot 58:3091-3097

Izawa T, Takahashi Y, Yano M (2003) Comparative biology comes into bloom: genomic and genetic comparison of flowering pathways in rice and Arabidopsis. Curr Opin Plant Biol 6:113-120

Kojima S, Takahashi Y, Kobayashi Y, Monna L, Sasaki T et al (2002) $H d 3 a$, a rice ortholog of the Arabidopsis FT gene, promotes 
transition to flowering downstream of $H d l$ under short-day conditions. Plant Cell Physiol 43:1096-1105

Kojima Y, Ebana K, Fukuoka S, Nagamine T, Kawase M (2005) Development of an RFLP-based rice diversity research set of germplasm. Breed Sci 55:431-440

Komiya R, Yokoi S, Shimamoto K (2008) Hd3a and RFT1 are essential for flowering in rice. Development 135:767-774

Komiya R, Yokoi S, Shimamoto K (2009) A gene network for longday flowering activates $R F T 1$ encoding a mobile flowering signal in rice. Development 136:3443-3450

Kosambi DD (1944) The estimation of map distance from recombination values. Ann Eugen 12:172-175

Lander ES, Green P, Abrahamson J, Barlow A, Daly MJ et al (1987) Mapmaker: an interactive computer package for constructing primary genetic linkage maps of experimental and natural populations. Genomics 1:174-181

Lin HX, Ashikari M, Yamanouchi U, Sasaki T, Yano M (2002) Identification and characterization of a quantitative trait locus, $H d 9$, controlling heading date in rice. Breed Sci 52:35-41

Lin HX, Liang ZW, Sasaki T, Yano M (2003) Fine mapping and characterization of quantitative trait loci $H d 4$ and $H d 5$ controlling heading date in rice. Breed Sci 53:51-59

Maas LF, McClung A, McCouch SR (2010) Dissection of a QTL reveals an adaptive, interacting gene complex associated with transgressive variation for flowering time in rice. Theor Appl Genet 120:875-1071

Matsubara K, Kono I, Hori K, Nonoue Y, Ono N et al (2008a) Novel QTLs for photoperiodic flowering revealed by using reciprocal backcross inbred lines from crosses between japonica rice cultivars. Theor Appl Genet 117:935-945

Matsubara K, Yamanouchi U, Wang ZX, Minobe Y, Izawa T et al (2008b) Ehd2, a rice ortholog of the maize INDETERMINATE1 gene, promotes flowering by up-regulating Ehdl. Plant Physiol 148:1425-1435

McCouch SR, Teytelman L, Xu Y, Lobos KB, Clare K et al (2002) Development and mapping of 2240 new SSR markers for rice (Oryza sativa L.). DNA Res 9:199-207

Murray MG, Thompson WF (1980) Rapid isolation of high molecular weight plant DNA. Nucl Acids Res 8:4321-4325

Nonoue Y, Fujino K, Hirayama Y, Yamanouchi U, Lin SY et al (2008) Detection of quantitative trait loci controlling extremely early heading in rice. Theor Appl Genet 116:715-722

Park SJ, Kim SL, Lee S, Je BI, Piao HL et al (2008) Rice Indeterminate 1 (OsId1) is necessary for the expression of Ehdl (Early heading date 1) regardless of photoperiod. Plant $\mathrm{J}$ 56:1018-1029

Takahashi Y, Shomura A, Sasaki T, Yano M (2001) Hd6, a rice quantitative trait locus involved in photoperiod sensitivity, encodes the $\alpha$ subunit of protein kinase CK2. Proc Natl Acad Sci USA 98:7922-7927

Takahashi Y, Teshima KM, Yokoi S, Innan H, Shimamoto K (2009) Variations in Hd1 proteins, $H d 3 a$ promoters, and Ehd1 expression levels contribute to diversity of flowering time in cultivated rice. Proc Natl Acad Sci USA 106:4555-4560

Takai T, Nonoue Y, Yamamoto S, Yamanouchi U, Matsubara K et al (2007) Development of chromosome segment substitution lines derived from backcross between indica donor rice cultivar 'Nona Bokra' and japonica recipient cultivar 'Koshihikari'. Breed Sci 57:257-261

Tamaki S, Matsuo S, Wong HL, Yokoi S, Shimamoto K (2007) Hd3a protein is a mobile flowering signal in rice. Science 316:1033-1036

Temnykh S, Park WD, Ayres N, Cartinhour S, Hauck N et al (2000) Mapping and genome organization of microsatellite sequences in rice (Oryza sativa L.). Theor Appl Genet 100:697-712

Tsuji H, Tamaki S, Komiya R, Shimamoto K (2008) Florigen and the photoperiodic control of flowering in rice. Rice 1:25-35

Uga Y, Nonoue Y, Liang ZW, Lin HX, Yamamoto S et al (2007) Accumulation of additive effects generates a strong photoperiod sensitivity in the extremely late-heading rice cultivar 'Nona Bokra'. Theor Appl Genet 114:1457-1466

Xue W, Xing Y, Weng X, Zhao Y, Tang W et al (2008) Natural variation in Ghd7 is an important regulator of heading date and yield potential in rice. Nat Genet 40:761-767

Yamamoto T, Kuboki Y, Lin SY, Sasaki T, Yano M (1998) Fine mapping of quantitative trait loci, $H d-1, H d-2$ and $H d-3$, controlling heading date of rice, as single Mendelian factors. Theor Appl Genet 97:37-44

Yamamoto T, Lin HX, Sasaki T, Yano M (2000) Identification of heading date quantitative trait locus $H d 6$ and characterization of its epistatic interactions with $H d 2$ in rice using advanced backcross progeny. Genetics 154:885-891

Yamamoto T, Yonemaru J, Yano M (2009) Towards the understanding of complex traits in rice: substantially or superficially? DNA Res 16:141-154

Yamane H, Ito T, Ishikubo H, Fujisawa M, Yamagata H et al (2009) Molecular and evolutionary analysis of the Hd6 photoperiod sensitivity gene within Genus Oryza. Rice 2:56-66

Yano M, Katayose Y, Ashikari M, Yamanouchi U, Monna L et al (2000) Hdl, a major photoperiod sensitivity quantitative trait locus in rice, is closely related to the Arabidopsis flowering time gene CONSTANS. Plant Cell 12:2473-2483

Yano M, Kojima S, Takahashi Y, Lin HX, Sasaki T (2001) Genetic control of flowering time in rice, a short-day plant. Plant Physiol 127:1425-1429

Yonemaru J, Yamamoto T, Fukuoka S, Uga Y, Hori K et al (2010) Q-TARO: QTL annotation rice online database. Rice 3:194-203 